\title{
Etude jurisprudentielle de la liquidation du régime matrimonial des époux pour cause de mort face à la succession
}

\author{
Par KISIMBA BONDO Kis, KABOZYA WA KABOZYA Joseph et MUBAKA \\ KATALAY Véro*
}

\section{INTRODUCTION}

Il y a un temps pour tout, un temps pour naître, et un temps pour mourir, c'est-à-dire que la vie et la mort sont deux réalités lorsqu'elle intervient, elle produit certaines conséquences juridiques à la naissance (mariage et décès) par exemple.

C'est ainsi que le droit ayant une place de choix dans toute société humaine, il apparait comme régulateur des relations et de l'évolution de la vie de l'homme. Cette idée est vérifiable dans tous les domaines de l'homme lors de la conclusion du mariage, il y a les effets patrimoniaux et extrapatrimoniaux qui donnent naissance à un régime matrimonial des époux.

Cependant, nous découvrons que la vie de l'homme est éphémère, c'est-à-dire celui-ci n'est pas éternel et à son décès il y aura ouverture de la succession, mais bien avant cela il faut d'abord liquider son régime matrimonial s'il était marié. La succession, elle consiste à transférer les biens d'une personne pour cause de mort à ses héritiers, par contre la liquidation du régime matrimonial consiste elle, à identifier les biens du de cujus et du conjoint survivant mettant ainsi fin à la copropriété et à la cogestion du patrimoine commun.

Le problème de successions ne nous épargne pas et s'impose à tout homme et tout l'homme, cela de près ou de loin. Ainsi dans la ville de Lubumbashi plusieurs conflits sont pendant dans des juridictions, car au décès d'une personne les membres de sa famille viennent s'imposer sur l'ensemble du patrimoine, privant ainsi le droit des héritiers de la première catégorie et du conjoint survivant qui lui doit premièrement bénéficier des biens du fait de la liquidation de leur régime matrimonial qui venait de prendre fin au décès de l'autre conjoint. Nous constatons une violation criante de la loi qui organise la succession et la fin d'un régime matrimonial.

Face à cette réalité des choses, plusieurs préoccupations sont venues à l'esprit et dont l'essentiel est coulé en cette problématique :

* Tous Assistants à la Faculté de Droit de l’Université de Lubumbashi. 
- Quel est le sort d'une succession qui n'a pas connue la liquidation du régime matrimonial des époux?

- En outre, nous remarquons dans la suite de la lecture des jugements que les juges n'apportent pas d'importance à la liquidation du régime matrimonial, est-ce que c'est par ignorance ou de manière délibérée?

En terme des réponses, il sied de noter que, sur ce qui est du sort d'une succession qui n'a pas connue la liquidation du régime matrimonial des époux, nous estimons que cette succession est frappée de nullité, car elle n'a pas respectée la procédure normale qui consiste avant tout à liquider le régime matrimonial des époux pour éviter la confusion des biens du conjoint survivant et du de cujus ou encore éviter que les biens du conjoint survivant soient liquider aussi au même titre que ceux du de cujus. Nous soulignons ici que la succession est possible que pour cause de mort et porte uniquement sur les biens seul du de cujus.

Il est de bon droit que toute succession de la personne qui été mariée soit précédée par la liquidation de leur régime matrimonial pour éviter la confusion des patrimoines. Comme le souligne le professeur KIFWABALA TEKILAZAYA Jean Pierre que : « la liquidation du régime matrimonial consiste en une opération tendant à établir le patrimoine du conjoint survivant et du de cujus, identifier les créances qui doivent être payées avant tout partage du patrimoine commun $\gg .{ }^{1}$

Sur ce qui est de l'ignorance ou mieux de la délibération du propre chef des juges quant à la liquidation du régime matrimonial des époux lors du décès d'un conjoint.

Certains juges estiment que la liquidation du régime matrimonial est un accessoire et la succession le principal, et donc pour eux la liquidation du régime matrimonial des époux pour cause de mort doit s'effectuée après toutes les opérations de la succession.

Par contre certains estiment aussi que la liquidation du régime matrimonial des époux pour cause de mort est réglée de façon tacite, c'est-à-dire de manière informelle car, rare sont des personnes qui saisissent les tribunaux pour cette question.

Soulignons en outre que la succession ne porte pas sur le patrimoine commun des époux, mais sur le patrimoine distinct des époux. Il sied de noter que le jugement qui liquide la succession avant le régime matrimonial des époux n'a pas de valeur juridique, car il viole une étape importante, celle de liquider avant tout le régime matrimonial des époux pour cause de mort, question de préserver les intérêts du conjoint survivant.

\section{A. DE LA SUCCESSION}

\section{Définition}

De manière générale, le terme succession aurait plusieurs sens, selon qu'il s'agit de la coutume et selon qu'il s'agit du droit.

1 KIFWALABA TEKILAZAYA JP, Droit civil congolais, régimes matrimoniaux, successions et libéralités, P.U.L., Lubumbashi, 2013, p.37. 
Dans le terme purement coutumier, le mot succession signifierait, remplacer une personne à son poste, soit à sa femme ou à son mari. Il est vrai que cette conception des choses par la coutume est d'ordre extrapatrimonial, alors que le droit extrapatrimonial est lié à la qualité de la personne et cesse au moment du décès.

Du point de vue juridique, le législateur congolais n'a pas donné une définition dans le code de la famille titre 1 du livre 4 qui dans son entièreté parle des successions et qui comportent 64 articles à travers lesquels l'on ne trouve aucune définition nous semble être proposée, la succession peut être définie comme : «l'ensemble des biens qu'une personne laisse à sa mort et que les héritiers légaux ou testamentaires et autres légataires récupèrent soit de par les dispositions légales ou de par les testaments du de cujus. ${ }^{2}$

Il y a lieu de retenir aussi que : « la succession est une cession des biens d'une personne (que nous appelons ici invariablement, le de cujus ou le défunt) pour cause de mort aux héritiers afin que ces derniers désormais le relais et continuent ainsi à l'assurer dans son actif et son passif même auprès des tiers avec lesquels, il avait des engagements ${ }^{3}{ }^{3}$

Il en résulte de ces deux définitions sus-évoquées, que la succession est le fait de transférer les biens d'une personne à ses héritiers pour cause de mort. Il se dégage que la succession traite uniquement du patrimoine net du de cujus (conjoint décédé et non pas de l'ensemble du patrimoine des époux).

\section{OUVERTURE DE LA SUCCESSION}

On appelle ouverture de la succession, « le fait qui entraine la dévolution des biens d'une personnes à ses héritiers $\gg{ }^{4}$ En effet, au décès d'une personne plusieurs interrogations peuvent être posées, quelles personnes habilitées à recueillir la succession, la qualité des héritiers et leurs capacités, ainsi que le lieu d'ouverture de celle-ci.

Il faut cependant, souligner que la succession est ouverte au domicile de la personne, après réunion des éléments nécessaires selon le cas d'une personne a une autre (la mort, l'absence, la disparition).

\section{DESIGNATION DU LIQUIDATEUR}

Le principe étant ainsi consacré par la loi et par la jurisprudence, l'article 795 du code de la famille dispose que : « en cas de succession Ab intestat, le plus âgé des héritiers sera chargé de la liquidation de la succession ou en cas de désistement, celui qui sera désigné par les héritiers $\gg .{ }^{5}$

2 MUPILA NDJIEKE HF, Les successions en droit congolais, pax-Congo, Kinshasa, 2000.

3 KAMBELE KUYUYILA, Comment gérer l'héritage familial, Médiaspaul, Kinshasa, 2010.

4 DEKKERS RENE, Précis de droit civil belge, Tome 3, les régimes matrimoniaux, les successions, les donations et les testaments, Bruyaut, Bruxelles, 1995.

5 Article 795 de la loi No87-010 portant code de la famille, du 01 Aout 1987. 
La volonté de la législation est que le liquidateur désigné fasse parti des héritiers de la $1^{\text {er }}$ catégorie et qu'il soit le plus âgé de la dite catégorie. Rappelons que dans cette catégorie nous avons des enfants nés dans et hors mariage affiliés par leur père géniteur de son vivant et les enfants adoptés tous sont héritiers de la $1^{\text {er }}$ catégorie sur un même pied d'égalité et jouissent des mêmes droits ${ }^{6}$. Déjà la charte de l'ONU et la constitution congolaise (allègue que) tous sommes égaux devant la loi départ notre naissance, homme et femme, fille et garçon.

Le liquidateur est choisi par le conseil de famille à l'unanimité et ce dit conseil soumet sa volonté à la juridiction compétente qui approuve leur choix par un jugement. Qu'il y a des cas ou le liquidateur désigné est un enfant né d'un autre lit nous assistons généralement que ce conjoint survivant et les enfants du $1^{\text {er }}$ lit ne facilite pas le liquidateur a accompli les taches qui sont mis à sa charge tel que l'énumère l'article 797 du code de la famille livre III de la famille, et arrive aussi qu'il soit même victime des menaces. Dans ce genre de cas qu'il sera préférable que le liquidateur puissent faire prévaloir ces droits afin de se sécuriser car selon la volonté du législateur en cas de contestation pour une cause valable les héritiers peuvent initier une action en contestation contre le liquidateur où la juridiction compétente, ensuite proposer un autre liquidateur est que la juridiction leur accorde un autre liquidateur dit légal avec des exigences. Si non ces genres de comportements doivent être sanctionnés par l'Etat d'autant plus que c'est par la volonté de famille qu'il a été désigné.

Dans l'affaire, RS 2391/IV succession Edwine ILUNGA WA NKULU décédé à Lubumbashi le 14/08/2010. Le conseil de famille réuni ont désigné MUKALAY MWAMBA Fils ainé, et vu les dispositions du code de la famille il a été confirmé comme liquidateur. Une décision du tribunal de paix Lubumbashi KAMALONDO, puisque ayant réuni toutes les conditions y afférentes. ${ }^{7}$

Dans le RS 2006, succession MIKUWA WA MULANGU décédé à Lubumbashi, après conseil de famille, Madame KALALA MULAJI a été choisie liquidatrice de ladite succession dont deux enfants sont mineurs d'âge.

Le tribunal estime favorable de confirmé KALALA MULAJI en qualité de liquidatrice, mais sous réserve de liquider les biens qu'au moment où les deux enfants seront majeurs. ${ }^{8}$

De la jurisprudence abondante, l'on s'accorde que les juges confirment les choix des familles qui portent sur les ainés d'office comme liquidateur.

\section{EXCEPTION}

L'alinéa 5 de l'article 795 du code de la famille dispose que : "Lorsque les héritiers ne sont pas encore connus ou sont trop éloignés, ou qu'ils ont tous renoncé à l'hérédité ou en cas de

6 Article 758 de la loi $n^{\circ} 16 / 008$ du 15 juillet 2016modifiant et complétant la loi $n^{\circ} 87-010$ portant code de la famille.

7 TRIPAIX/L'SHI-KAMALONDO, RS 2391, 10 MARS 2011.

8 TRIPAIX/L'SHI-KAMALONDO, RS 2006, Du 20 Juin 2000. 
constations graves sur la liquidation, le tribunal compétent désigne d'office ou à la requête du ministère public ou d'un des héritiers, un liquidateur judiciaire parent ou étranger à la famille $»{ }^{9}$

Il en résulte qu'à l'absence des héritiers de la première catégorie, les héritiers des autres catégories ou une personne étrangère à la succession (famille) peuvent être liquidateurs, une désignation faite par le tribunal.

Le tribunal de grande instance de Lubumbashi dans l'affaire, RS 2476 dans le conflit succession KABWE ODI, ou tous les héritiers ont renoncé à la succession, par simple fait qu'ils étaient en désaccords avec leur père avant son décès.

$\mathrm{Vu}$ l'urgence et sur demande du ministère public, le tribunal avait choisi le grand frère $\mathrm{du}$ de cujus et le conjoint survivant comme Co-liquidateur de ladite succession. ${ }^{10}$

Mais le liquidateur ainsi désigné, n'est pas tenu d'accepter les fonctions qui lui sont confiées, il peut se démettre en évoquant des justes motifs. ${ }^{11}$

\section{LES ROLES DU LIQUIDATEUR DANS UNE SUCCESSION}

Le rôle du liquidateur dans une succession est celui d'un gérant et d'un administrateur, après son installation il doit procéder à l'identification des biens des époux pour faire la séparation entre les biens du de cujus sur lesquels devra s'ouvrir la succession et les biens du conjoint survivant selon leur régime matrimonial, il doit aussi identifier les héritiers.

Cette étape est la plus importante et constitue même le rôle complet du liquidateur. Après avoir identifié les biens du conjoint survivant et du de cujus, ainsi que les héritiers qui doivent bénéficier de la succession du de cujus, le liquidateur sera dans l'obligation de faire un rapport détailler aux héritiers sur quoi sera fonder le partage successoral.

Il faut souligner que le liquidateur «Agit, non pas en son nom, encore moins en son propre compte, mais au nom et pour le compte des héritiers pour lesquels il est mandaté. Il en résulte qu'il ne peut pas confondre son propre patrimoine avec celui de la succession. ${ }^{12}$

\section{B. DE LA LIQUIDATION DES REGIMES MATRIMONIAUX DES EPOUX POUR CAUSE DE MORT}

Le décès d'un conjoint met fin au régime matrimonial, car l'on ne peut pas être dans une copropriété avec un mort. C'est ainsi qu'au décès d'un conjoint l'examen de leur régime matrimonial est souhaitable pour éviter une confusion des biens d'un vivant et d'un mort.

9 Article 795 code de la famille, alinéa 5.

10 TGI/L'SHI, RS 2476, 27 Avril 2000.

11 KIFWABALA TEKILAZAYA JP., op.cit., p.40.

12 KAMBALE KUYUSILA, op.cit., p.28. 
« La liquidation est l'ensemble des opérations tendant à établir la constance des actifs et passifs, et à identifier les créanciers à payer avant le partage du patrimoine commun, mais aussi à faire la répartition des biens du conjoint survivant et du conjoint décédé. ${ }^{13}$

\section{DU REGIME DE LA SEPARATION DES BIENS}

Il est paradoxal de parler de la liquidation du régime de la séparation des biens étant donné que ledit régime ne comporte pas de communauté. ${ }^{14} \mathrm{Il}$ n'est donc pas un régime à liquider mais un régime qui s'achève. ${ }^{15}$ Mais à cause des incertitudes des preuves des confusions d'intérêts et de l'augmentation du nombre des indivisions soumises aux règles générales de partages ${ }^{16}$ la liquidation est donc incontestable.

Dans la séparation des biens chaque époux a ses propres biens, mais le but recherché est celui d'éviter toute confusion lorsqu'un époux (survivant) n'est pas à mesure de prouver qu'un bien lui appartient. Il y va s'en dire que pendant la vie commune les époux sous la séparation des biens peuvent acheter ensemble certains biens, à dire vrai ils seront en indivision. Notons que les biens indivis font partie du domaine à liquider entre le conjoint survivant et le de cujus, ainsi le partage sera fait sur les biens indivis, et la succession portera sur les biens seul du de cujus et non pas sur l'ensemble des biens y compris les biens indivis.

De toute la littérature évoquée ci-haut, il y a lieu de noter que nos juridictions n'ont pas jusqu'à présent connue une affaire en liquidation du régime de la séparation des biens, parce que les époux n'en fait jamais cas lors de la célébration de leur mariage devant l'officier de l'état civil car considérer comme discriminatoire à l'égard de l'autre époux.

\section{DU REGIME DE LA COMMUNAUTE UNIVERSELLE}

Le régime de la communauté universelle a proprement il y a que les biens communs, par conséquent lors du décès d'un conjoint les biens communs seront partagés par deux. Ainsi le conjoint survivant reprendra sa part et la masse successorale sera constituée de l'autre moitié

La déduction est donc, la communauté universelle lors de la liquidation il ne se pose pas de problèmes, car de manière plus simple les biens sont partagés entre les deux conjoints (survivant et héritiers du de cujus). Mais d'aucuns n'ignorent que même dans ce régime, il ne manque pas de biens propres à chaque époux et donc par conséquent ses biens propres ne seront pas partagés.

13 KIFWABALA TEKILAZAYA JP, op.cit., p.178.

14 LIPISKI, La liquidation dans le régime de séparation de biens, LGDJ, Paris, 2002.

15 BEIGNIER BERNARD, Les régimes matrimoniaux, Mont chrétiens, Paris, 2008.

16 MALAURIE PHILIPPE ET AYNES LAURENT, Droit civil, les successions libéralités, CUJAN, Paris, 1998. 
Il se dégage ensuite que, ce régime consacre entre conjoint une copropriété sur tous les biens (meubles et immeubles) et une responsabilité commune quant aux dettes présentes et avenir. Il se dégage que ces dettes dites communes sont celles contractées par les époux dans l'intérêt supérieur du ménage. A ce sujet, lors du décès d'un conjoint ces dettes seront payées moitié par le conjoint survivant et moitié par la succession du de cujus.

C'est ainsi que le tribunal avait jugé dans le RC 2614; affaire en liquidation du régime matrimonial de la communauté universelle. Entre la veuve NGOIE KALOMBO demanderesse. La veuve, 22 ans plus tard, après la mort de son époux vient saisir le tribunal en liquidation de leur régime matrimonial; car estima être désavantagée dans le partage des biens par les frères du de cujus.

La veuve motive sa demande en s'appuyant sur le prescrit de l'article 503 du code de la famille.

Mais quant à la décision du tribunal, il se réserve de liquider le régime parce que la veuve n'avait pas la preuve de leur mariage et de leur régime. ${ }^{17}$

C'est ainsi que le tribunal dans le RC 2785, entre YUMA RAMAZANI demanderesse, saisi le tribunal pour liquider son régime matrimonial, car depuis la mort de son époux on n'avait pas fait cette opération.

Le juge saisi ce dit réservé pour liquider le régime matrimonial, pour raisons non avancé par lui. ${ }^{18}$

C'est ainsi que le tribunal avait jugé dans le RC 2580, affaire en liquidation du régime matrimonial, Madame KASONGO LYDIE saisi un mois après le décès de son mari pour que la liquidation de leur régime matrimonial soit faite et que les biens soient partagés par moitié, parce que les frères du de cujus sont de mauvaise foi $\mathrm{a}-\mathrm{t}$ - elle indiquée.

Le tribunal constata que le de cujus était marié à une dame décédée bien avant lui, et que celle qui vient demander la liquidation c'est la deuxième femme mariée aussi légalement après la mort de la première femme.

$\mathrm{Vu}$ l'importance de la matière, le tribunal ce dit réservé quant à liquider le régime matrimonial, parce que le premier mariage n'avait pas connu de liquidation. ${ }^{19}$

Mais pour le deux premier jugement, les juges n'ont pas motivés leurs argumentaires sur le refus de la liquidation des régimes matrimoniaux pour permettre l'ouverture de manière précise de la succession.

\section{DU REGIME DE LA COMMUNAUTE REDUITE AUX ACQUETS}

Avant de liquider le régime de la communauté réduite aux acquêts, il faut déterminer quels sont les biens propres et les acquêts du conjoint survivant et du de cujus. A ce sujet le conjoint survivant reprend ses biens propres et les autres propres pour le ce cujus.

17 TRIPAIX/L'SHI-KAMALONDO, RC 2614, 5 Juin 2008.

18 TRIPAIX/L'SHI-KAMALONDO, RC 2785, 4 Septembre 2012.

19 TRIPAIX/L'SHI-KAMALONDO, RC 2614, 5 Juin 2008. 
Ainsi donc la liquidation se fera par moitié sur les acquêts et non pas sur les biens propres des époux.

C'est ainsi que le tribunal avait jugé sous l'affaire RC 2735 KIBAMBE NGONGO décédé depuis maintenant une année, ordonnant au liquidateur d'identifier les créanciers communs, les héritiers et les biens propres, de procéder directement et immédiatement au paiement des dettes communes et ensuite partagés les biens restant par deux, une partie au conjoint survivant et à la succession du de cujus. ${ }^{20}$

Nous louons la décision du tribunal car, ayant respecté les prescrits de l'article $503 \mathrm{du}$ code de la famille, fort est notre étonnement car cette liquidation a été sollicité du tribunal par un homme cas rare dans notre société.

Toutefois, il y a des biens qui échappent aux règles régissant la liquidation du régime matrimonial. Ainsi le tribunal de PAIX RWASHI/KAMPEMBA avait jugé qu'une société créée par les époux n'est pas régie par les mêmes règles régissant la liquidation du régime matrimonial. Il y a là une entité juridique différente de celles des associés, un patrimoine propre ne se confondant pas aux patrimoines des associés. ${ }^{21}$

Il va s'en dire que, en matière de liquidation du régime matrimonial des époux pour cause de mort, peu de jugement ont été tranchés par les tribunaux, il est ainsi regrettable pour les juges de liquider la succession ignorant, la liquidation du régime matrimonial pour éviter les confusions des biens.

\section{CONCLUSION}

Comme la mort est inévitable pour tous celui qui respirent encore, la succession, qui s'ensuit, comme mode de transmission du patrimoine d'une personne à ses héritiers et autres légataires doit être précédée par la liquidation de son régime matrimonial si elle était mariée.

Toutefois, eu égard à la passion qui couvre cet évènement douloureux qu'est la mort le règlement de la succession mérite bien qu'on l'aborde avec sérieux surtout pour celui qui était marié, car du fait que ce sont les biens qui sont en jeux.

Certains praticiens du droit ignorent la liquidation du régime matrimonial car considéré comme accessoire et la succession le principal, est donc pour eux la liquidation du régime matrimonial doit s'effectuer après toutes les opérations de la succession, par contre ils estiment que la liquidation du régime matrimonial est réglée de façon tacite, c'est-à-dire de manière informelle.

Cependant, une succession bien réglée est celle précédée par la liquidation du régime matrimonial, car l'objectif est d'éviter que même les biens du conjoint survivant soient confondus à la masse successorale. Mais dans la lecture des jugements rares sont des cas ou les juges ont liquidé les régimes matrimoniaux des époux, violant ainsi les prescrits de la 
loi. Nous pensons qu'il est de bon droit et pour l'intérêt supérieur du conjoint survivant, qu'avant de liquider la succession soit liquider d'abord le régime matrimonial, pour éviter de faire succéder même les biens d'un vivant, alors qu'il y a succession que pour cause de mort et celle-ci porte seulement sur les biens du de cujus.

\section{BIBLIOGRAPHIE}

\section{TEXTE DES LOIS}

1. La loi $N^{\circ} 87-010$ portant code de la famille, du 01 Aout 1987, modifiée par la loi $n^{\circ} 16 / 008$ du 15 Juillet 2016.

\section{LA JURISPRUDENCE}

1. RS 2391, du 10 Mars 2011

2. $R S 2006$, du 20 Juin 2000

3. RS 2476, du 27 Avril 2000

4. $R C 2615$, du 05 Juin 2008

5. $R C$ 2785, du 04 Septembre 2012

6. $R C 2963$, du 10 Mars 2014

7. $R C 2735$, du 28 Juin 2012

8. RC 5971, du 11 Mai 2009

\section{LES OUVRAGES}

1. BEIGNIER BERNARD, Les régimes matrimoniaux, Montchrestien, Paris, 2008

2. DEKKERS RENE, Précis de droit civil belge, Tome 3, les régimes matrimoniaux, les successions, les donations et les testaments, Bruyant, Bruxelles, 1995

3. KAMBALE KUYUSILA, comment gérer l'héritage familial, Médiaspaul, Kinshasa, 2010

4. KIFWABALA TEKILAZAYA JP., Droit civil congolais, Régimes matrimoniaux, successions et libéralités, PUL, Lubumbashi, 2013.

5. LIPISKI, La liquidation dans le régime de la séparation des biens, LGDJ, Paris 2002

6. MALAURIE PHILIPPE et AYNES LAURENT, Droit civil, les successions, libéralités, Cujas, Paris, 1998

7. MUPILA NDJIEKE HP, Les successions en droit congolais, Kinshasa, Pax-Congo, 2000. 